\title{
Differential alterations in mitochondrial function induced by a choline-deficient diet: Understanding fatty liver disease progression ${ }^{\text {th }}$
}

\author{
João S. Teodoro ${ }^{a}$, Anabela P. Rolo ${ }^{a}$, Filipe V. Duarte ${ }^{a}$, Anabela M. Simões ${ }^{a}$, Carlos M. Palmeira ${ }^{\mathrm{a}, \mathrm{b}, *}$ \\ ${ }^{a}$ Center for Neurosciences and Cell Biology, Department of Zoology, University of Coimbra, 3004-517 Coimbra, Portugal \\ ${ }^{\mathrm{b}}$ IMAR, Mitochondrial Research Group, Department of Zoology, University of Coimbra, 3004-517 Coimbra, Portugal
}

\section{A R T I C L E I N F O}

\section{Article history:}

Received 26 March 2008

Received in revised form 3 July 2008

Accepted 30 July 2008

Available online 15 August 2008

\section{Keywords:}

Fatty liver

Mitochondria

Oxidative phosphorylation

Adenine nucleotide translocator

Mitochondrial permeability transition

\begin{abstract}
A B S T R A C T
Non-alcoholic fatty liver disease (NAFLD) is an increasingly reported pathology, characterized by fat accumulation within the hepatocyte. Growing evidences suggest specific effects on mitochondrial metabolism, but it is still unclear the relationship between fatty liver progression and mitochondrial function. In the present work we have investigated the impact of fatty liver on mitochondrial bioenergetic functions and susceptibility to mitochondrial permeability transition (MPT) induction in animals fed a choline-deficient diet (CDD) for 4, 8, 12 or 16 weeks. Mitochondria isolated from CDD animals always exhibited higher state 4 respiration. Mitochondrial membrane potential was decreased in CDD animals at 4 and 16 weeks. At 12 weeks, oxidative phosphorylation was more efficient in CDD animals, suggesting a possible early response trying to revert the deleterious effect of increased triglyceride storage in the liver. However, mitochondrial dysfunction was evident in CDD animals at 16 weeks as indicated by decreased $\mathrm{RCR}$ and $\mathrm{ADP} / \mathrm{O}$, with a corresponding decrease in respiratory chain enzymes activities. Such loss of respiratory efficiency was associated with accumulation of protein oxidation products, in tissue and mitochondrial fraction. Additionally, although no differences in ATPase activity, the lag phase was increased in mitochondria from CDD animals at 16 weeks, associated with decreased content of the adenine nucleotide translocator. Increased susceptibility to calcium-induced MPT was evident in CDD animals at all time points. These results suggest a dynamic mechanism for the development of NALFD associated with altered mitochondrial function.
\end{abstract}

(ㄷ) 2008 Elsevier B.V. and Mitochondria Research Society. All rights reserved.

\section{Introduction}

Non-alcoholic fatty liver disease (NAFLD) is an increasing modern world disease, recognized as the basis for inflammatory hepatitis and induction of liver cirrhosis (Clark and Diehl, 2003). It is marked by the accumulation of fat, mainly in the form of free fatty acids and triglycerides, inside the hepatocytes (Pessayre et al., 2002; Diraison et al., 2003). This pathology may be caused by increased dietary fat consumption (an increasingly common phenomenon, especially in developed countries) or due to deficiencies in any of the steps of lipid metabolism. A deregulation in fatty acid's metabolism leads to an overproduction of triglycerides with subsequent fat accumulation inside the liver cells. The excessive abundance of free fatty acids causes a shift in metabo-

\footnotetext{
Work partially supported by FCT (PTDC/SAU-OSM/72443/2006). A.P. Rolo, J.S. Teodoro and F.V. Duarte are recipients of a fellowship from Science and Technology Foundation (SFRH/BPD/26514/2006, SFRH/BD/38467/2007 and SFRH/ $\mathrm{BD} / 38372 / 2007$, respectively).

* Corresponding author. Address: IMAR, Mitochondrial Research Group, Department of Zoology, University of Coimbra, 3004-517 Coimbra, Portugal. Tel.: +351239 855760; fax: +351239855789.

E-mail address: palmeira@ci.uc.pt (C.M. Palmeira).
}

lism, turning these molecules into the main energy source of the cell (Haque and Sanyal, 2002; McClain et al., 2004). Such alteration increases lipid peroxidation, destabilizing the inner cell redox environment (Rao and Reddy, 2001).

The choline-deficient diet (CDD) model for fatty liver disease, relates with the fact that choline is essential for the correct assembly and export of the very low density lipoproteins (VLDLs), the main vehicle of fat exportation from the liver to the peripheral adipose tissue. Being unable to correctly produce and export VLDLs, the CDD-fed animals develop fat accumulation in the liver (Koteish and Diehl, 2001).

Being the main ATP production source for the cell (where almost $90 \%$ of the ATP is produced), mitochondria play a key role in the majority of metabolic pathways, including a central role in lipid metabolism (Schatz, 1995). It is one of the most susceptible organelles for damage with a profound involvement in the onset of diverse pathologies (Mahler et al., 1997; Jacobsen et al., 2001; Brealey et al., 2002; Sayeed, 2002; DiMauro and Schon, 2003; Duchen, 2004). Indeed, progressive decline of mitochondrial function correlates with disease progression. An example is microvesicular steatosis, which occurs in conditions characterized by severe impairment of the mitochondrial $\beta$-oxidation due to genetic and/ or acquired causes (Fromenty and Pessayre, 1997). 
Previous work has demonstrated that fat accumulation is severely impairing to mitochondrial function (Leclercq et al., 2000; Caldwell et al., 2004; Douette et al., 2005; Begriche et al., 2006; Teodoro et al., 2006). However, the progression of the pathology has never been reported. As so, we hypothesize that excessive fat accumulation in hepatocytes will impair their mitochondrial bioenergetics, in a cause-effect relationship, in the CDD model. This could supply several pieces of essential information for the further understanding of the disease's onset and progression towards more aggressive pathological situations. By analyzing several parameters of proved relevance we demonstrate that there is an apparent response mechanism of the liver to fat accumulation. This response can revert almost all of the deleterious effects caused by the accumulation of fat. However, this is a transient and frail mechanism, since the continuous aggression caused by the fat destroyed the capacity of response of the organ, which ultimately was associated to, amongst other factors, a severe depletion on the mitochondrial content on adenine nucleotide translocator (ANT). This loss of ANT could be a cause for the observed loss of oxidative phosphorilative efficiency.

\section{Materials and methods}

\subsection{Animals}

Seven-weeks-old male Wistar rats (Charles River, France) were divided in two groups of 24 animals and housed under controlled light and humidity conditions with free access to food and water. The control diet (\#118754) and choline-deficient diet (\#118753) were obtained from Dyets Inc., Bethlehem, PA, USA. Animals were sacrificed after $4,8,12$ or 16 weeks of feeding with control or choline-deficient diet. The animals were weighted immediately before sacrifice and killed by cervical dislocation and decapitation.

\subsection{Materials}

All compounds were purchased from Sigma-Aldrich Chemical Co. (St. Louis, MO). All other reagents and chemicals used were of the highest grade of purity commercially available.

\subsection{Liver histology}

Livers from the animals were quickly and immediately removed after the animal sacrifice, fixed in $10 \%$ buffered formaline and stained with haematoxylin and eosin (H\&E) (Palmeira et al., 2003).

\subsection{Preparation of liver mitochondria}

Mitochondria were isolated from the liver of the Wistar rats by conventional methods (Gazotti et al., 1979), with slight modifications (Palmeira and Wallace, 1997). Protein content was determined by the biuret method (Gornall et al., 1949), calibrated with bovine serum albumin.

\subsection{Evaluation of plasma biochemical parameters}

Right after its sacrifice, a sample of the animal's blood was collected, centrifuged at $2300 \mathrm{~g}$ for $3 \mathrm{~min}$. Plasma was analyzed with commercial kits.

\subsection{Determination of hepatic triglyceride content}

The measurement of the hepatic triglyceride content was performed using a commercially available triglyceride detection kit (Linear Chemicals, Spain). Briefly, $50 \mathrm{mg}$ of liquid nitrogen freezethawed hepatic tissue were homogenized in $1 \mathrm{~mL}$ isopropanol and immediately centrifuged for $5 \mathrm{~min}$ at $2300 \mathrm{~g}$. Supernatant
$(10 \mu \mathrm{L})$ was added to a $1 \mathrm{~mL}$ of a kit-supplied reagent and incubated at $37{ }^{\circ} \mathrm{C}$ for $5 \mathrm{~min}$. The absorbance was measured at $500 \mathrm{~nm}$ and the quantification occurred by comparison with a kit-supplied standard.

\subsection{Evaluation of mitochondrial oxygen consumption}

Oxygen consumption of isolated mitochondria was polarographically determined with a Clark oxygen electrode (Estabrook, 1967), as previously described (Palmeira et al., 1994). Mitochondria ( $1 \mathrm{mg}$ ) were suspended under constant stirring, at $25^{\circ} \mathrm{C}$, in $1 \mathrm{~mL}$ of standard respiratory medium (130 mM sucrose, $50 \mathrm{mM} \mathrm{KCl,} 5 \mathrm{mM}$

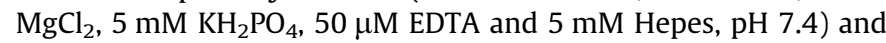
energized by adding glutamate/malate or succinate to a final concentration of $5 \mathrm{mM}$. For succinate assays, $2 \mu \mathrm{M}$ rotenone, an inhibitor of complex I, were previously added. State 3 respiration was induced by adding ADP. Oxygen consumption was also measured in the presence of oligomycin $(0.5 \mu \mathrm{g} / \mathrm{mg}$ protein) and $1 \mu \mathrm{M}$ carbonylcyanide-p-trifluoromethoxyphenylhydrazon (FCCP).

\subsection{Evaluation of mitochondrial membrane potential $(\Delta \Psi)$}

$\Delta \Psi$ was estimated using an ion-selective electrode to measure the distribution of tetraphenylphosphorium $\left(\mathrm{TPP}^{+}\right)$according to previously established methods (Palmeira et al., 1994; Kamo et al., 1979). Mitochondria ( $1 \mathrm{mg}$ ) were suspended in $1 \mathrm{~mL}$ of standard respiratory medium (as in mitochondrial respiration) supplemented with $3 \mu \mathrm{M} \mathrm{TPP}{ }^{+}$. A matrix volume of $1.1 \mu \mathrm{L} / \mathrm{mg}$ was assumed.

\subsection{Determination of enzymatic activities}

ATPase activity was determined spectrophotometrically at $660 \mathrm{~nm}$, in association with ATP hydrolysis. The reaction was carried out at $37^{\circ} \mathrm{C}$, in $2 \mathrm{~mL}$ reaction medium ( $125 \mathrm{mM}$ sucrose, $65 \mathrm{mM} \mathrm{KCl}, 2.5 \mathrm{mM} \mathrm{MgCl}$ and $5 \mathrm{mM}$ Hepes, $\mathrm{pH} \mathrm{7.4).} \mathrm{After} \mathrm{the}$ addition of freeze-thawed mitochondria $(0.25 \mathrm{mg})$, the reaction was initiated by adding $2 \mathrm{mM} \mathrm{Mg}^{2+}$-ATP, in the presence or absence of oligomycin ( $1 \mu \mathrm{g} / \mathrm{mg}$ protein). After $10 \mathrm{~min}$, adding $1 \mathrm{~mL}$ of $40 \%$ trichloroacetic acid stopped the reaction. Samples were centrifuged for $5 \mathrm{~min}$ at $3000 \mathrm{~g}$ and $2 \mathrm{~mL}$ of ammonium molybdate

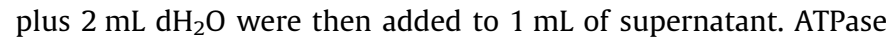
activity was calculated as the difference in total absorbance and absorbance in the presence of oligomycin.

Succinate-dehydrogenase activity was polarographically determined as previously described (Singer, 1994). The reaction was carried out at $25^{\circ} \mathrm{C}$ in $1.3 \mathrm{~mL}$ of standard respiratory medium (as in mitochondrial respiration) supplemented with $5 \mathrm{mM}$ succinate, $2 \mu \mathrm{M}$ rotenone, $0.1 \mu \mathrm{g}$ antimycin $\mathrm{A}, 1 \mathrm{mM} \mathrm{KCN}$ and $0.3 \mathrm{mg}$ Triton $\mathrm{X}-100$. After the addition of freeze-thawed mitochondria $(0.25 \mathrm{mg})$, the reaction was initiated by adding $1 \mathrm{mM}$ phenazinemetasulphate (PMS).

Cytochrome $c$ oxidase activity was polarographically determined, as previously described (Brautigan et al., 1978). The reaction was carried out at $25^{\circ} \mathrm{C}$ in $1.3 \mathrm{~mL}$ of standard respiratory medium (as in mitochondrial respiration) supplemented with $2 \mu \mathrm{M}$ rotenone, $10 \mu \mathrm{M}$ oxidized cytochrome $c$ and $0.3 \mathrm{mg}$ Triton $\mathrm{X}-100$. After the addition of freeze-thawed mitochondria $(0.25 \mathrm{mg})$, the reaction was initiated by adding $5 \mathrm{mM}$ ascorbate plus $0.25 \mathrm{mM}$ tetramethylphenylene-diamine (TMPD).

\subsection{Determination of carbonyl group content}

Carbonyl group content in both isolated mitochondria and hepatic tissue was measured spectrophotometrically by using 2,4dinitrophenyl-hydrazine (DNPH) (Levine et al., 1990; Schild et al., 
1997). Briefly, $100 \mathrm{mg}$ of fresh tissue were homogenized in $1 \mathrm{~mL}$ of lysis buffer (250 mM sucrose, $0.5 \mathrm{mM}$ EGTA and $10 \mathrm{mM}$ Hepes, $\mathrm{pH}$ 7.4 , supplemented with protease inhibitors). Ice-cold trichloroacetic acid $(20 \% \mathrm{w} / \mathrm{v}, 500 \mu \mathrm{L})$ was added to mitochondria $(1 \mathrm{mg})$ or tissue homogenate $(50 \mu \mathrm{L})$ and allowed to stand for $15 \mathrm{~min}$ at $4{ }^{\circ} \mathrm{C}$. The precipitated protein was collected by centrifugation at $6500 \mathrm{~g}$ for $10 \mathrm{~min}$, and the supernatant was discarded. The pellet was fully resuspended in $500 \mu \mathrm{L}$ of $0.1 \mathrm{M} \mathrm{NaOH}$ and $500 \mu \mathrm{L}$ of $10 \mathrm{mM}$ DNPH dissolved in $2 \mathrm{M} \mathrm{HCl}$ were added to the samples. In parallel, a blank was prepared by addition of $500 \mu \mathrm{L}$ of $2 \mathrm{M} \mathrm{HCl}$ containing no DNPH to the protein sample. After incubation at room temperature, in the dark for $30 \mathrm{~min}, 500 \mu \mathrm{L}$ of trichloroacetic acid $(20 \% \mathrm{w} / \mathrm{v})$ were added to the samples. The precipitated protein was collected by centrifugation at $6500 \mathrm{~g}$ for $10 \mathrm{~min}$, and the supernatant was discarded. The pellet was washed three times with $1 \mathrm{~mL}$ of a $1: 1$ (vol/vol) mixture of ethanol and ethyl acetate and centrifuged at $6500 \mathrm{~g}$ for $5 \mathrm{~min}$ to remove the free reagent. The final precipitated protein was redissolved in $200 \mu \mathrm{L}$ of $6 \mathrm{M}$ guanidine hydrochloride solution. The amount of protein dinitrophenylhydrozone derivative was quantified by measuring the absorbance at $365 \mathrm{~nm}$ and converted to nmol of hydrazone by using a molar absorption coefficient of $22,000 \mathrm{M}^{-1} \mathrm{~cm}^{-1}$. Carbonyl content was expressed as nmol of DNPH incorporated per mg of protein.

\subsection{Western blotting analysis}

Frozen mitochondrial pellets were homogenized in lysis buffer (10 mM Tris- $\mathrm{HCl}, \mathrm{pH} 6.8,0.8 \% \mathrm{w} / \mathrm{v}$ SDS, $4 \% \mathrm{v} / \mathrm{v}$ glicerol, $2 \% \mathrm{v} / \mathrm{v} 2-$ mercaptoethanol and $0.5 \% \mathrm{w} / \mathrm{v}$ bromophenol blue) at $100^{\circ} \mathrm{C}$, for $5 \mathrm{~min}$. Aliquots were fractionated in $8 \%$ polyacrylamide gels, transferred to polyvinylidene difluoride membranes (Hybond ${ }^{\mathrm{m}}-\mathrm{P}$ ) and blotted with goat anti-ANT (1:10,000 dilution, sc9299 from Santa Cruz, CA, USA), goat anti-UCP-2 (1:2500 dilution, sc-6526 from Santa Cruz, CA, USA), goat anti- $\mathrm{F}_{1}-$ ATPase (1:5000 dilution, sc16689 from Santa Cruz, CA, USA) or rabbit anti-VDAC (1:5000 dilution, Ab3434 from AbCam, UK). The immuno-reactive proteins were visualized with bovine anti-goat IgG alkaline phosphatase conjugated (1:20,000 dilution, sc-2351 from Santa Cruz, CA, USA) and goat anti-rabbit IgG alkaline phosphatase conjugated (1:20,000 dilution, sc-2007 from Santa Cruz, CA, USA). Membranes were reacted with the ECF detection system (Amersham) and read with the VersaDoc imaging system (Bio-Rad Laboratories, Inc., CA, USA). The densitometric analysis was performed with the Quantity One software (Bio-Rad Laboratories, Inc., CA, USA).

\subsection{Detection of mitochondrial permeability transition induction}

The induction of the mitochondrial permeability transition(MPT) was evaluated spectrophotometrically at $540 \mathrm{~nm}$ by the determination of the mitochondrial swelling and consequent light scattering diminishing by calcium stimuli (Palmeira and Wallace, 1997). Mitochondria ( $1 \mathrm{mg}$ ) was added to $2 \mathrm{~mL}$ of reaction medium ( $200 \mathrm{mM} \mathrm{su}-$ crose, $1 \mathrm{mM} \mathrm{KH}_{2} \mathrm{PO}_{4}, 5 \mathrm{mM}$ succinate, $10 \mu \mathrm{M}$ EGTA, $3 \mu \mathrm{M}$ rotenone and $10 \mathrm{mM}$ Tris-Mops, $\mathrm{pH} 7.4$ ) to begin the reaction, at $25^{\circ} \mathrm{C}$. After a brief period, several calcium concentrations were added. In a selected tube, $1 \mu \mathrm{M}$ cyclosporin A (CyA) was added to serve as control.

\subsection{Citrate synthase assay}

Citrate synthase activity was determined spectrophotometrically at $412 \mathrm{~nm}$ at $25^{\circ} \mathrm{C}$, as previously described (Srere, 1969).

\subsection{Statistical analysis}

Data are presented as means \pm SEM of experiments with four different animals in each group. A Student's $t$-test was used for the comparison between animals fed a control or choline-deficient diet for the same number of weeks. A $P$ value $<0.05$ was considered statistically significant.

\section{Results}

\subsection{Characterization of the animal model}

Animals fed a choline-deficient diet (CDD animals) for 4, 8, 12 or 16 weeks presented an increase in body weight, when compared to control animals, except after 12 weeks of feeding. However, livers from CDD animals were heavier than control animals (Table 1 ) during all the time of the study, being statistically significant for 4 and 16 weeks. This is probably due to triglyceride accumulation, as shown in Fig. 1. The analysis of the biochemical plasma parameters did not reveal any alteration on the content on cholesterol, triglycerides or any of the evaluated liver enzymes in the blood of the analyzed animals (data not shown).

No specific histological alterations were observed in the livers from control animals. In contrast, under light microscope, sections stained with H\&E in the livers from the CDD group showed steatosis (Fig. 2). While the 4,8 and 12 weeks livers only presented severe macrovesicular steatosis, 16 weeks livers presented macro and microvesicular steatosis, which may suggest an impending change in the behaviour of the pathology. However, no inflammation markers were found, confirming the presence of steatosis alone.

No significant variation in the activity of citrate synthase, a mitochondrial enzymatic marker, was found between mitochondrial preparations from control and CDD animals (data not shown).

\subsection{Effects of fat accumulation on mitochondrial transmembrane potential}

Taking into account the fundamental role of mitochondrial transmembrane potential $(\Delta \Psi)$ for the phenomenon of oxidative phosphorylation, $\Delta \Psi$ was evaluated in isolated liver mitochondria, after energization with glutamate/malate or succinate. Comparatively to mitochondria isolated from control animals, 4 weeks of feeding with CDD caused a decrease in $\Delta \Psi$ in mitochondria energized with either glutamate/malate or succinate (Table 2). ADP-induced depolarization was decreased in succinate-energized mitochondria isolated from CDD animals. Furthermore, the mitochondrial capacity to establish $\Delta \Psi$ after ADP phosphorylation (repolarization) was also impaired in CDD animals, at 4 weeks. Interestingly, no significant changes were observed in liver mitochondria isolated from 8 weeks CDD-fed animals, suggesting a possible improvement of mitochondrial function (data not shown). In fact, $\Delta \Psi$ developed by mitochondria isolated from 12 weeks CDD animals, as well as ADP-induced depolarization and $\Delta \Psi$ after repolarization, were similar to mitochondria from control animals (Table 2 and Fig. 3A). However, after 16 weeks of feeding with CDD, a decrease in $\Delta \Psi$ in mitochondria energized with both glutamate/ malate and succinate was observed (Table 2 and Fig. 3B). Also,

Table 1

Liver weight in control and animals fed a choline-deficient diet (CDD)

\begin{tabular}{llrlr}
\hline & 4 weeks & 8 weeks & 12 weeks & 16 weeks \\
\hline Control & $15.5 \pm 0.96$ & $14.5 \pm 0.65$ & $16.3 \pm 1.25$ & $15.5 \pm 0.5$ \\
CDD & $24 \pm 0.91^{*}$ & $21 \pm 1.92$ & $21.3 \pm 3.01$ & $21 \pm 1.08^{*}$ \\
\hline
\end{tabular}

Values are expressed as means $\pm \operatorname{SEM}(n=4)$ from animals fed for $4,8,12$ or 16 weeks with a normal (control) or a choline-deficient diet (CDD). Values statistically significantly different from control for each week: ${ }^{*} P<0.05$. 


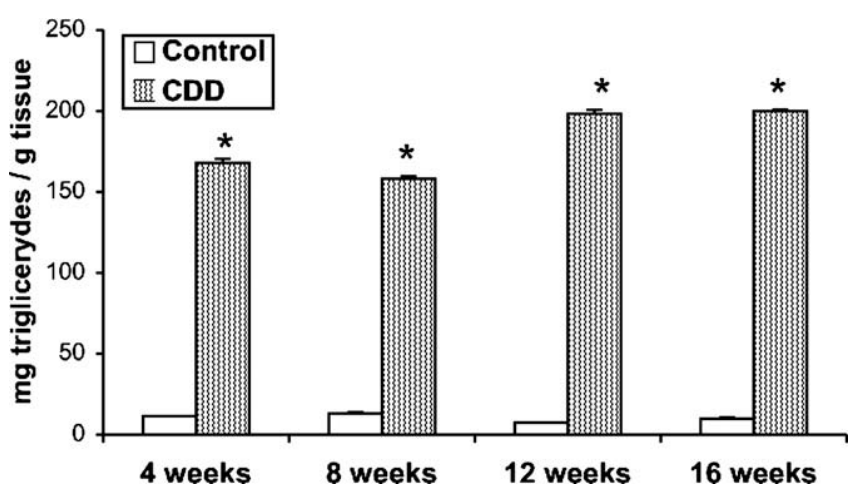

Fig. 1. Total amount of intrahepatic triglyceride content in control and animals fed a choline-deficient diet (CDD). Data expressed as means \pm SEM of four animals in each group. Values statistically significant different from control, for each week: " $P<0.05$. mitochondria from 16 weeks CDD-fed animals displayed impaired ability to recover membrane potential after ADP phosphorylation.

The lag phase (time necessary for ADP phosphorylation) was significantly affected by fat accumulation in 16 weeks CDD-fed animals (Fig. 3C), when compared with the lag phase evaluated in mitochondria from control animals. Interestingly, 12 weeks CDD animals presented a decreased lag phase, when compared to the same age control animals (Fig. 3C).

\subsection{Effects of fat accumulation on mitochondrial respiration}

Oxidative phosphorylation capacity of mitochondria isolated from control and steatotic animals was investigated by following oxygen consumption upon glutamate/malate or succinate oxidation.

Mitochondrial respiratory state 3 (consumption of oxygen in the presence of substrate and ADP) was decreased in mitochondria isolated from 4 and 16 weeks CCD-fed animals (Fig. 4A) irrespective of the substrate used. Similarly, oxygen consumption in the

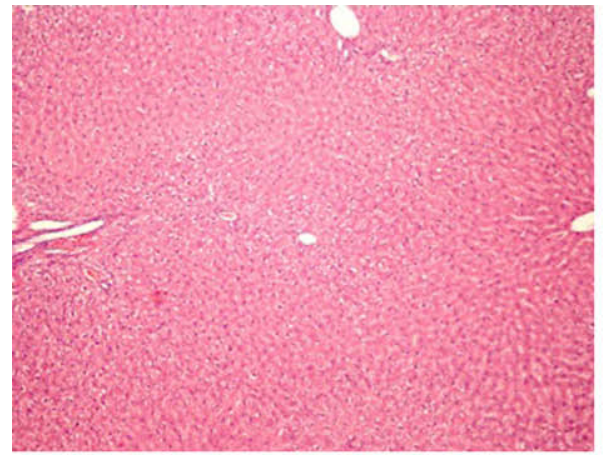

\section{Control 4 weeks 100x}

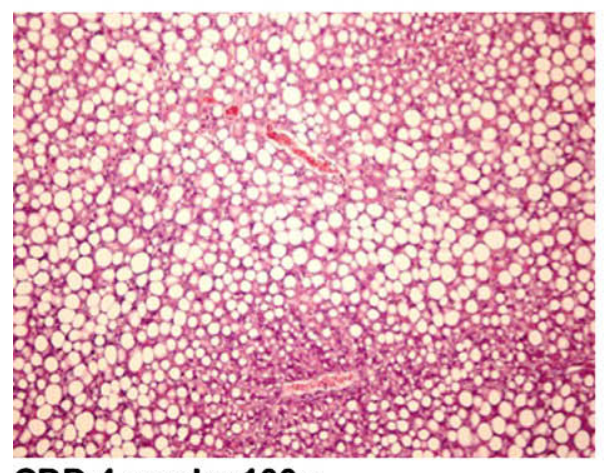

CDD 4 weeks 100x

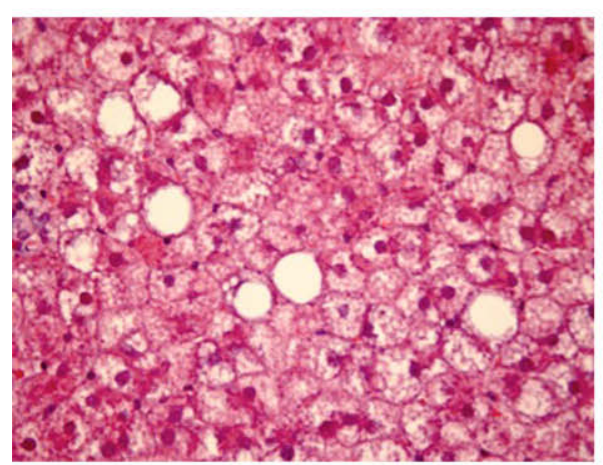

CDD 16 weeks $400 x$

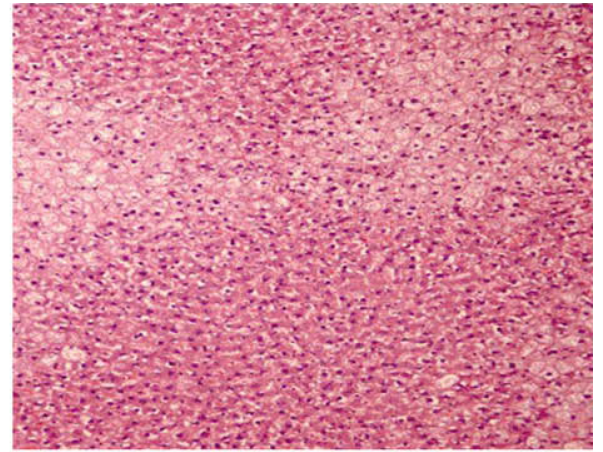

\section{Control 16 weeks $100 x$}

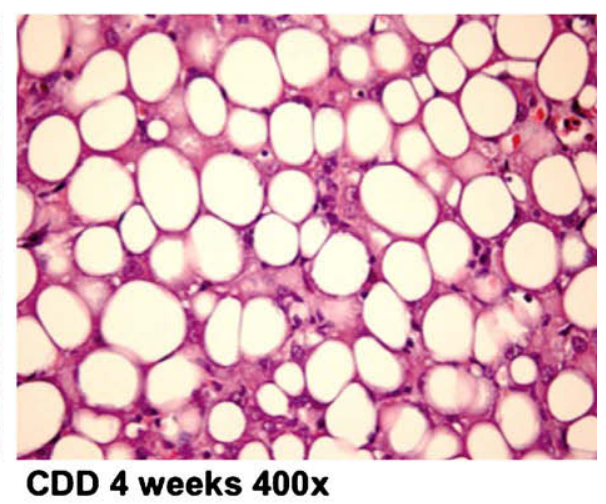

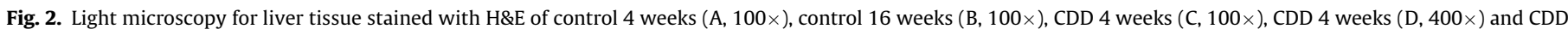
16 weeks $(\mathrm{E}, 100 \times)$ 
Table 2

Mitochondrial membrane potential $(\Delta \Psi)$ in control and animals fed for 4,12 and 16 weeks a choline-deficient diet (CDD)

\begin{tabular}{|c|c|c|c|c|c|}
\hline & & \multicolumn{2}{|c|}{ Glutamate/malate } & \multicolumn{2}{|l|}{ Succinate } \\
\hline & & Control & CDD & Control & CDD \\
\hline 4 weeks & $\begin{array}{l}\Delta \Psi(-\mathrm{mV}) \\
\text { Depolarization }(-\mathrm{mV}) \\
\text { Repolarization }(-\mathrm{mV}) \\
\text { Lag phase }(\mathrm{s})\end{array}$ & $\begin{aligned} 206.67 & \pm 0.41 \\
25.2 & \pm 0.53 \\
202.96 & \pm 0.4 \\
57 & \pm 0.48\end{aligned}$ & $\begin{aligned} 201 & \pm 0.51^{*} \\
23.31 & \pm 0.64 \\
195.38 & \pm 0.52^{*} \\
50.5 & \pm 0.48\end{aligned}$ & $\begin{array}{r}206.62 \pm 0.38 \\
39.98 \pm 0.43 \\
204.15 \pm 0.44 \\
123 \pm 0.83\end{array}$ & $\begin{array}{r}198.21 \pm 0.49^{*} \\
32.6 \pm 0.53^{*} \\
194.28 \pm 0.53^{*} \\
131 \pm 0.71\end{array}$ \\
\hline 12 weeks & $\begin{array}{l}\Delta \Psi(-\mathrm{mV}) \\
\text { Depolarization }(-\mathrm{mV}) \\
\text { Repolarization }(-\mathrm{mV}) \\
\text { Lag phase }(\mathrm{s})\end{array}$ & $\begin{aligned} 213.61 & \pm 0.29 \\
25.49 & \pm 0.26 \\
209.72 & \pm 0.4 \\
44.25 & \pm 0.91\end{aligned}$ & $\begin{array}{r}215.02 \pm 0.23 \\
23.39 \pm 0.28 \\
212.36 \pm 0.34 \\
36.75 \pm 0.57\end{array}$ & $\begin{array}{r}215.8 \pm 0.23 \\
36.26 \pm 0.26 \\
213.73 \pm 0.26 \\
112 \pm 0.67\end{array}$ & $\begin{aligned} 216.75 & \pm 0.18 \\
31.96 & \pm 0.19 \\
215.70 & \pm 0.2 \\
72 & \pm 0.57^{*}\end{aligned}$ \\
\hline 16 weeks & $\begin{array}{l}\Delta \Psi(-\mathrm{mV}) \\
\text { Depolarization }(-\mathrm{mV}) \\
\text { Repolarization }(-\mathrm{mV}) \\
\text { Lag phase }(\mathrm{s})\end{array}$ & $\begin{array}{r}216.76 \pm 0.14 \\
25.13 \pm 0.36 \\
213.97 \pm 0.16 \\
40.13 \pm 0.22\end{array}$ & $\begin{array}{r}209.54 \pm 0.28^{*} \\
17.45 \pm 0.33^{*} \\
199.88 \pm 0.42^{*} \\
55.5 \pm 0.43^{*}\end{array}$ & $\begin{array}{r}215.18 \pm 0.15 \\
32.17 \pm 0.26 \\
213.86 \pm 0.18 \\
90.5 \pm 0.48\end{array}$ & $\begin{array}{r}209.24 \pm 0.21^{*} \\
31.85 \pm 0.24 \\
201.93 \pm 0.29^{*} \\
129.5 \pm 0.56^{*}\end{array}$ \\
\hline
\end{tabular}

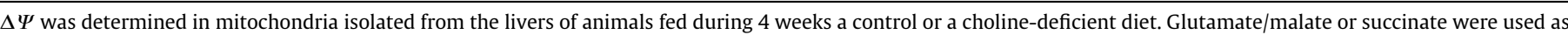

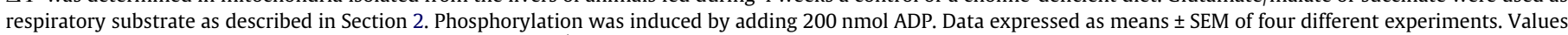
statistically significant different from control for each week: ${ }^{*} P<0.05$.

presence of FCCP, a well-known respiratory chain uncoupler, was also decreased in CDD animals after 4 and 16 weeks of feeding. No statistical significant alterations were observed in 8 weeks
CDD-fed animals. However, after 12 weeks, CDD animals exhibited increased state 3 and FCCP-induced respiratory rates (Fig. 4A). The $\mathrm{ADP} / \mathrm{O}$ ratio, an indicator of oxidative phosphorylation efficiency,
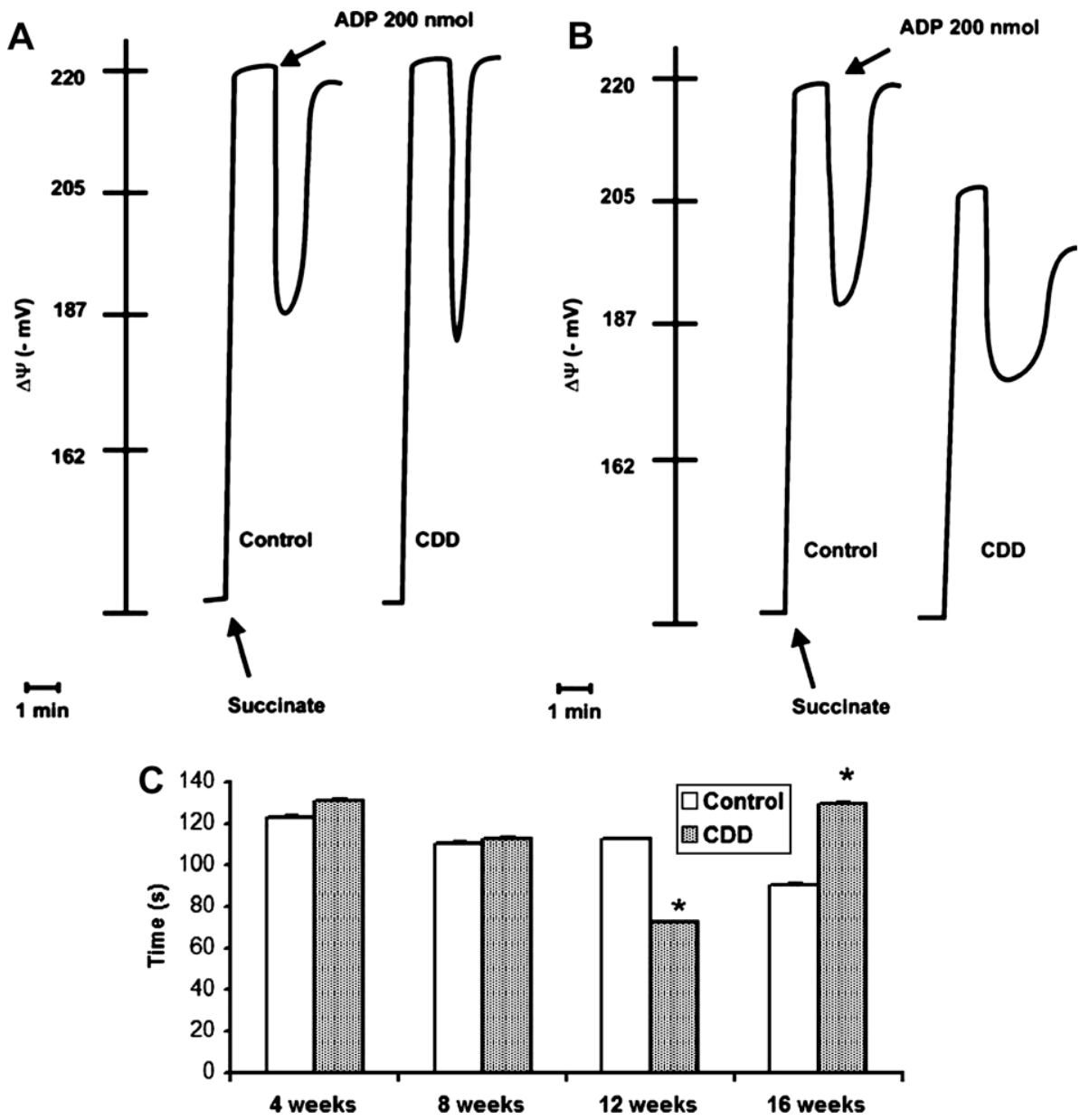

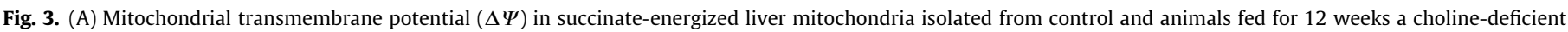

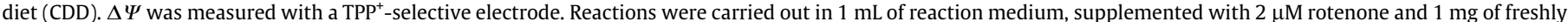

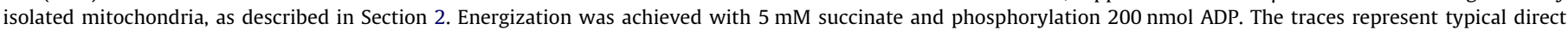

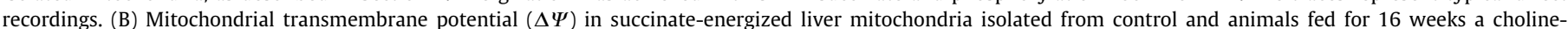

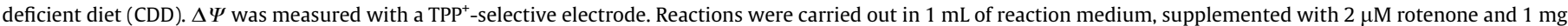

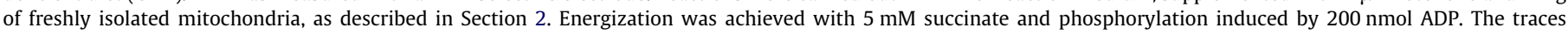

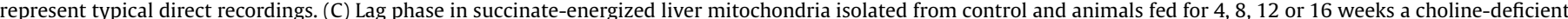

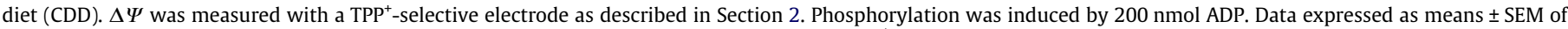
four different experiments. Values statistically significant different from control for each week: ${ }^{*} P<0.05$. 
was decreased in CDD animals after 4, 8 and 16 weeks of feeding, irrespective of the substrate used (data not shown), but it was similar to controls in mitochondria isolated from 12 weeks CDD-fed animals.

Fat accumulation induced a significant increase in mitochondrial respiratory state 4 (consumption of oxygen after ADP phosphorylation) in animals fed with CDD for $4,8,12$ or 16 weeks, when compared with state 4 of control animals, irrespective of the substrate used (Fig. 4B). The ratio between mitochondrial respiratory state 3 and state 4 (RCR) was significantly decreased in mitochondria isolated from 4,8 and 16 weeks animals fed with CDD, but not in 12 weeks CDD animals (data not shown).

Mitochondrial respiration in the presence of oligomycin, a known inhibitor of the mitochondrial $\mathrm{F}_{1} \mathrm{~F}_{\mathrm{o}}$-ATP synthase, was also increased in CDD animals at 4, 8, 12 and 16 weeks of feeding with CDD (Fig. 4B), suggesting an increased protonic leak (the passage of protons from the intermembrane space to the matrix without ATP synthesis).

\subsection{Effects of fat accumulation on mitochondrial enzyme activities and protein carbonyl content}

The decreased rate of oxygen consumption induced by FCCP in mitochondria isolated from 4 and 16 weeks CDD-fed animals, suggested alterations in the mitochondrial electron transport chain complexes. In view of this, succinate-dehydrogenase and cytochrome $c$ oxidase activities were evaluated. Mitochondria isolated from 16 weeks CDD-fed animals exhibited decreased activities in both of these enzymes, while at 12 weeks, activities of succinatedehydrogenase and cytochrome $c$ oxidase were increased, comparatively to the same age controls (Fig. $5 \mathrm{~A}$ and $5 \mathrm{~B}$, respectively). No statistical significant differences were observed at 4 and 8 weeks. Interestingly, the analysis of protein carbonyl content, a marker of protein oxidative damage, in both liver tissue and mitochondria, revealed an increased content of protein carbonyls in 16 weeks CDD-fed animals, but decreased in 12 weeks CDD animals, comparatively to controls (Table 3 ).

The decrease in the performance of phosphorylation of mitochondria isolated from CDD-fed animals for 4,8 and 16 weeks, as reflected by an increased lag phase, and decreased ADP/O, suggested alterations at the level of the mitochondrial $\mathrm{F}_{1} \mathrm{~F}_{\mathrm{o}}$-ATP synthase, a key component of the phosphorylative system. The evaluation of mitochondrial ATPase activity revealed a less efficient ATPase in
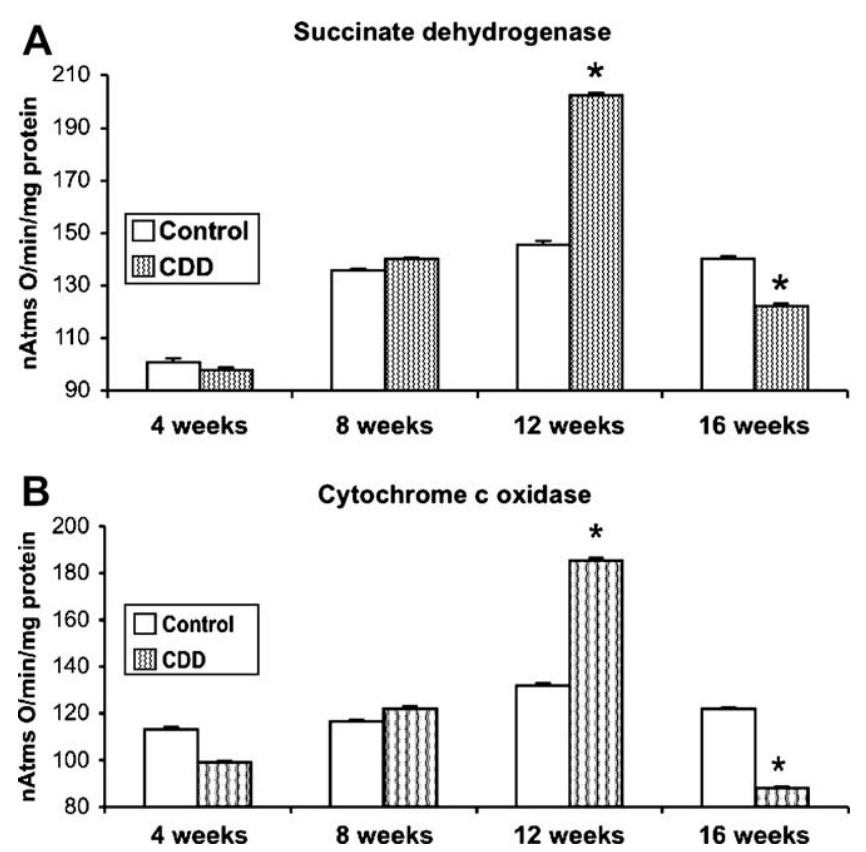

Fig. 5. (A) Succinate-dehydrogenase activity in control and animals fed a cholinedeficient diet (CDD) for 4, 8, 12 or 16 weeks. Enzyme activity was polarographically determined with a Clark oxygen electrode. Mitochondria $(0.25 \mathrm{mg})$ were suspended under constant stirring, at $25^{\circ} \mathrm{C}$, in $1 \mathrm{~mL}$ of standard respiratory medium. The reaction media was supplemented with $5 \mathrm{mM}$ succinate, $2 \mu \mathrm{M}$ rotenone, $0.1 \mu \mathrm{g}$ antimycin A, $1 \mathrm{mM}$ KCN, $0.3 \mathrm{mg}$ Triton X-100 and the reaction initiated by adding $1 \mathrm{mM}$ PMS. Data expressed as means \pm SEM of four different experiments. Values statistically significant different from control for each week: ${ }^{*} P<0.05$. (B) Cytochrome $c$ oxidase activity in animals fed a choline-deficient diet (CDD) for 4, 8, 12 or 16 weeks. Enzyme activity was polarographically determined with a Clark oxygen electrode. Mitochondria ( $0.25 \mathrm{mg}$ ) were suspended under constant stirring, at $25^{\circ} \mathrm{C}$, in $1 \mathrm{~mL}$ of standard respiratory medium. The reaction media was supplemented with $2 \mu \mathrm{M}$ rotenone, $10 \mu \mathrm{M}$ cytochrome $c, 1 \mathrm{mM} \mathrm{KCN}, 0,3 \mathrm{mg}$ Triton X-100 and the reaction initiated by adding $0.25 \mathrm{mM}$ ascorbate/TMPD. Data expressed as mean$\mathrm{S} \pm \mathrm{SEM}$ of four different experiments. Values statistically significant different from control for each week: ${ }^{*} P<0.05$.

mitochondria isolated from 8 weeks CDD-fed animals, when compared to mitochondria from control animals (Fig. 6). No differences statistically significant were observed at 4 and 16 weeks. However, mitochondria from 12 weeks CDD-fed animals exhibited an increase
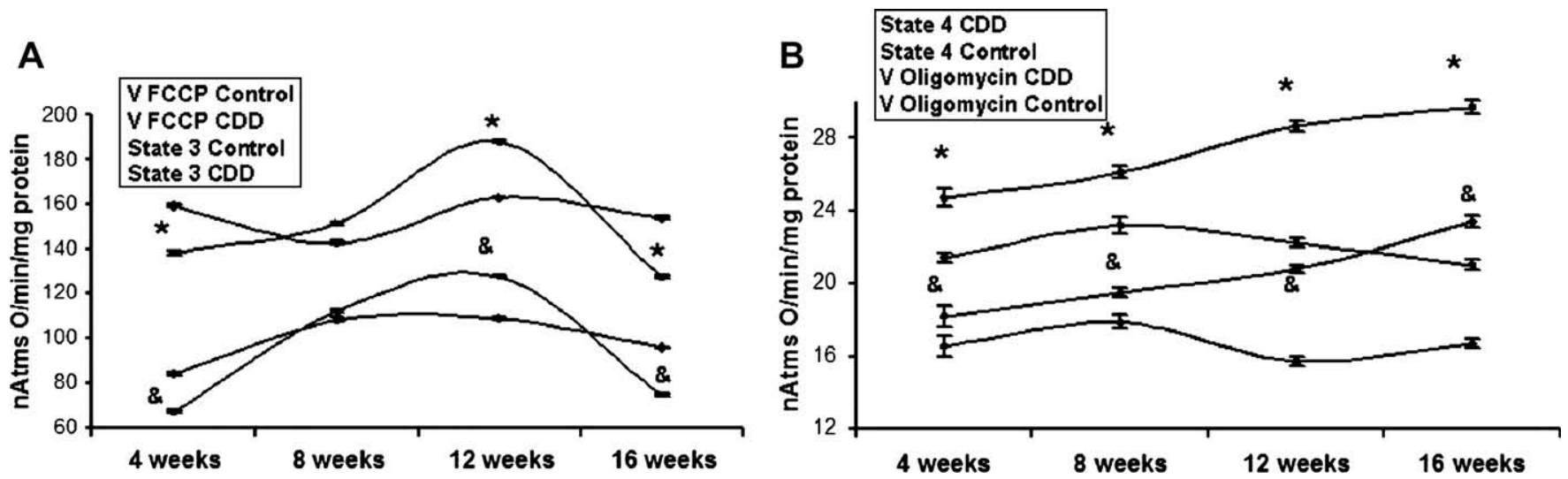

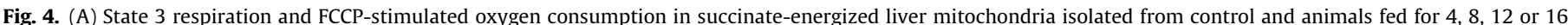

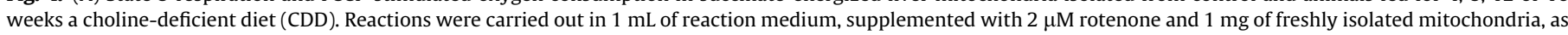

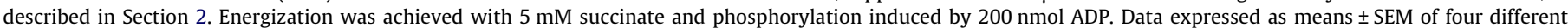

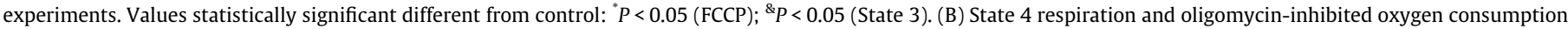

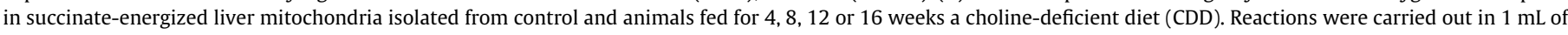

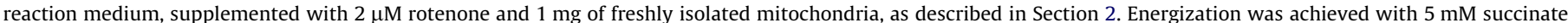

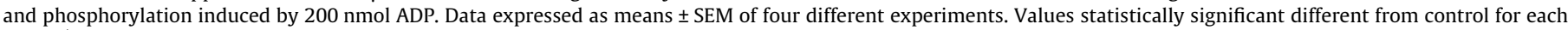
week: ${ }^{*} P<0.05$ (State 4 ); ${ }^{\&} P<0.05$ (oligomycin). 
Table 3

Carbonyl content in hepatic tissue and mitochondria from control and animals fed for 12 or16 weeks a choline-deficient diet (CDD)

\begin{tabular}{lrr}
\hline Sample & \multicolumn{1}{c}{ Control } & \multicolumn{1}{c}{ CDD } \\
\hline Tissue 12 weeks & $3.6 \pm 0.75$ & $2.4 \pm 1.18^{*}$ \\
Tissue 16 weeks & $2.3 \pm 0.31$ & $3.6 \pm 0.56^{*}$ \\
Mitochondria 12 weeks & $2.57 \pm 0.27$ & $1.15 \pm 0.65^{*}$ \\
Mitochondria 16 weeks & $1 \pm 0.34$ & $2.6 \pm 0.57^{*}$
\end{tabular}

Carbonyl group content in both isolated mitochondria and hepatic tissue was measured spectrophotometrically by using 2,4-dinitrophenyl-hydrazine (DNPH) as described in Section 2. Data expressed as means \pm SEM of four different experiments. Values statistically significant different from control, for each week: "P $<0.05$.

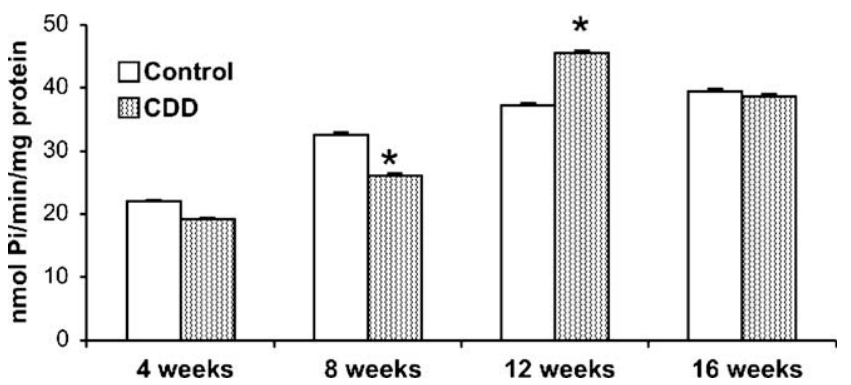

Fig. 6. ATPase activity in liver mitochondria isolated from control and animals fed for $4,8,12$ or 16 weeks a choline-deficient diet (CDD). ATPase activity was evaluated spectrophotometrically at $660 \mathrm{~nm}$, in association with ATP hydrolysis. Reactions were carried out in $2 \mathrm{~mL}$ of reaction medium, supplemented with $0.25 \mathrm{mg}$ of freeze-thawed mitochondria, as described in Section 2. Data expressed as means \pm SEM of four different experiments. Values statistically significant different from control for each week: ${ }^{*} P<0.05$.

in ATPase activity, when compared to control animals (Fig. 6), comparatively to the respective controls.

\subsection{Effects of fat accumulation on mitochondrial protein content}

Since the decrease in the phosphorylative efficiency induced by 16 weeks of feeding was not associated with impaired activity of the ATP synthase, the content on adenine nucleotide translocator (ANT), another component of the phosphorylative system, was evaluated. The content on ANT was found to be decreased after 16 weeks, but tending to be increased after 12 weeks of feeding (Fig. 7). Additionally, the increase in ATPase activity observed at 12 weeks was not associated with increased content, since no changes were observed comparatively to the respective control animals (Fig. 7).

The mitochondrial content in uncoupling protein UCP-2 was also evaluated, since CDD animals exhibited increased state 4 respiratory rates that were not caused by an increased leak through the ATP synthase, as indicated by the experiments with oligomycin. However, no changes were observed on the UCP-2 content (Fig. 7).

3.6. Effects of fat accumulation on the susceptibility to calciuminduced mitochondrial membrane permeability (MPT)

Mitochondria possess a finite capacity for accumulating calcium before undergoing the calcium-dependent mitochondrial permeability transition (MPT). Mitochondria isolated from 4, 8, 12 or 16 weeks CDD-fed animals, were more susceptible to undergo the calcium-dependent mitochondrial swelling, in comparison with the respective control animals (Fig. 8). Pretreatment with CyA completely prevented calcium-dependent mitochondrial swelling.

\section{Discussion}

The loss of mitochondrial functionality is undoubtedly associated with the emergence of several aggressive human pathologies (Mahler et al., 1997; Jacobsen et al., 2001; Brealey et al., 2002; Sayeed, 2002; DiMauro and Schon, 2003; Duchen, 2004). In the fatty liver, the accumulation of free fatty acids due to disturbances in lipid metabolism or due to diets with high-fat content (Haque and Sanyal, 2002) alters the energetic metabolism of the cell, with high rates of fatty acid oxidation that lead to mitochondrial impairment (Browning and Horton, 2004). Altered mitochondrial morphology was described early in an animal model of fatty liver
12 weeks
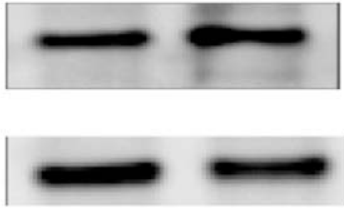
$F_{1}$-ATPase

UCP-2 (33 kDa)

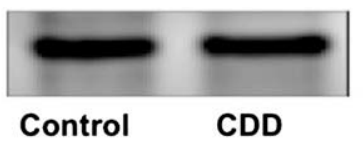

16 weeks
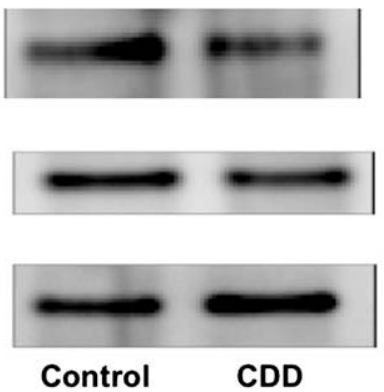

ANT/NDAC ( $\%$ Control)

CDD 12 weeks

$125.75 \pm 12.22$
CDD 16 weeks
$71.68 \pm 7.28^{\star}$

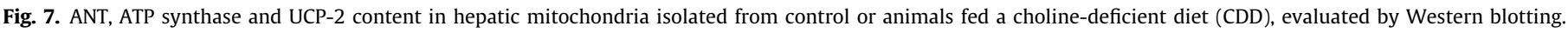

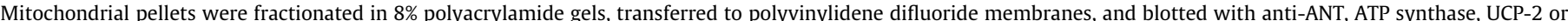

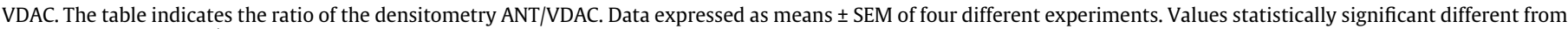
control for each week: ${ }^{*} P<0.05$. 


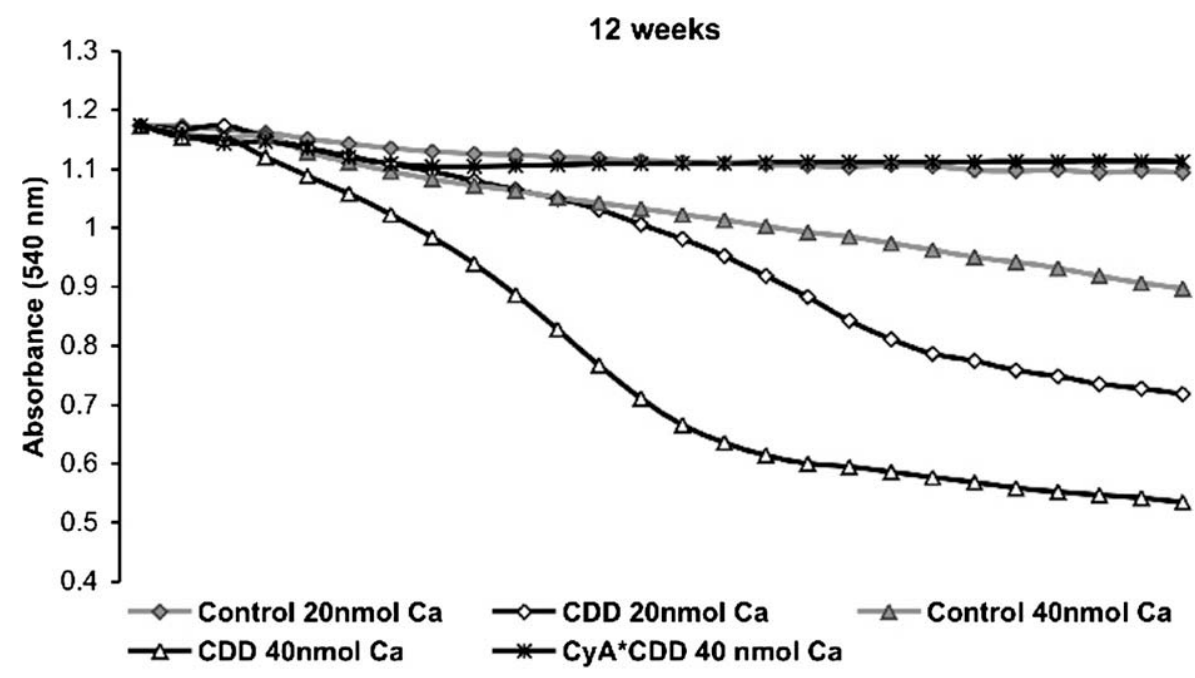

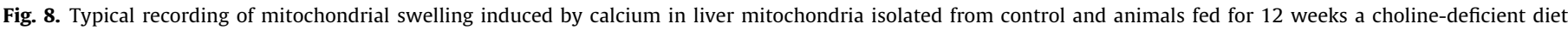

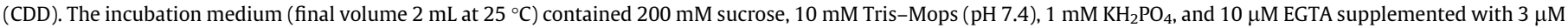

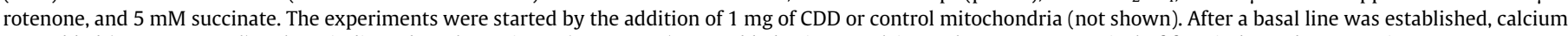
was added (20 or $40 \mathrm{nmol}$ ). Where indicated, cyclosporine A (CyA, $1 \mu \mathrm{M}$ ) was added prior to calcium. The traces are typical of four independent experiments.

(Danzani, 1957). From then on, several studies were conducted on diverse models of steatosis, where mitochondrial dysfunction was found and studied. However, the mechanisms underlying the link between impaired mitochondrial function and NAFLD onset still eludes the scientific community.

This paper further recognizes that fat accumulation in the liver is associated with decreased efficiency of the phosphorylative system, in concordance with our previous studies in another animal model of fatty liver (Teodoro et al., 2006). Although other studies have been conducted with the choline-deficient model of fatty liver disease (Vendemiale et al., 2001; Oliveira et al., 2002; Grattagliano et al., 2003; Zafrani, 2004), this work proved invaluable since it is the first of its kind to apply a timeline approach. The mitochondrial function, mainly at the bioenergetic level, was found to be impaired due to fat accumulation. Interestingly, these data showed that, apparently, there is an attempt, by the liver, to cope and recover from the fat insult, as shown by the data regarding 12 weeks CDD feeding.

CDD animals, at 4 and 16 weeks, exhibited decreased $\Delta \Psi$, when compared to control mitochondria. Respiratory state 4 was higher at $4,8,12$ and 16 weeks in mitochondria from CDD animals. Respiration, in the presence of oligomycin, an ATP synthase inhibitor, was stimulated in CDD animals, when compared to control. This suggested an increase in mitochondrial internal membrane permeability to protons, which was not associated with higher content of UCP- 2 content. Respiratory state 3 was decreased in CDD animals, at 4 and 16 weeks, but stimulated at 12 weeks, when compared to control animals. Similar results were observed for uncoupled respiration, suggesting alterations at the level of the electron transport chain at 16 weeks. Respiratory control ratio was decreased in mitochondria from CDD animals, at 4 and 16 weeks. The lag phase was lower in CDD animals at 12 weeks, but higher at 16 weeks in CDD animals, while $\mathrm{ADP} / \mathrm{O}$ ratio was decreased at 8 and 16 weeks, when compared to the respective controls. These observations suggest that at 12 weeks, there is a mechanism to stimulate the efficiency of the phosphorylative system, capacity that is abolished at 16 weeks. Mitochondrial ATPase activity was lower in CDD animals, at 8 weeks than in controls, but higher at 12 weeks, associated with decreased ATP/ADP ratio at 8 weeks and decreased ATP content at 16 weeks, but higher ATP/ADP ratio at 12 weeks. CDD animals at 16 weeks had a significant reduction in adenine nucleotide transloca- tor (ANT), probably the cause for the decreased efficiency of the phosphorylative system. In CDD animals, succinate-dehydrogenase and cytochrome $c$ oxidase activities were higher at 12 weeks, but lower at 16 weeks. Additionally, at 12 weeks, there was a decrease in carbonyl content in both hepatic tissue and mitochondria but at 16 weeks carbonyl content was higher in CDD animals than in control.

The analysis of the mitochondrial transmembrane potential $(\Delta \Psi)$ revealed that the capability of creating and maintaining a potential was compromised on mitochondria from CDD animals, after 4 and 16 weeks of feeding with a choline-deficient diet. This decreased $\Delta \Psi$ observed in CDD-fed animals could be the result of an increase in the permeability of the mitochondrial inner membrane to protons (proton leak). In fact, the resting mitochondrial oxygen consumption, the state 4 respiratory rate, was found to be significantly increased in CDD animals at 4, 8, 12 and 16 weeks, as well the respiratory velocity in the presence of oligomycin, an ATP synthase inhibitor. These data suggest an increased protonic leak, which can be in part attributed to membrane alterations caused by phosphatidylcholine deficiency, the main phospholipid of eukaryotic membranes (Zeisel and Blusztajn, 1994). No alterations in UCP-2 content were observed, thus ruling out the role of uncoupling proteins on the increased resting oxygen consumption. A causal link between UCP-2 induction and fatty liver disease is still not established. In fact, high-fat feeding of UCP-2 knockout animals had no effect on fatty liver disease progression (Baffy et al., 2002), suggesting that UCP-2 expression has no protective or deleterious effect on hepatic injury associated to fatty liver. Additionally, and similarly to our results, a recent study has shown that animals fed diets deficient in choline or methionine had no alterations in hepatic UCP-2 content (Donthamsetty et al., 2007).

The decrease in both state 3 respiratory rate and FCCP-induced oxygen consumption indicated a loss of oxidative capacity in mitochondria from CDD animals. Such impairment was probably caused by decreased efficiency of the mitochondrial electron transport chain, as demonstrated by the decrease in the activities of both succinate-dehydrogenase and cytochrome $c$ oxidase, in mitochondria from 16 weeks CDD-fed animals. Such alteration could be caused by oxidative damage, as the content in protein carbonyls was increased in both hepatic tissue and mitochondria of these animals. One of the biochemical mechanisms indicated as 
responsible for the evolution of NAFLD to more aggressive pathologies is the increased production of reactive oxygen species (ROS) (Pessayre et al., 2002; Begriche et al., 2006; Day and James, 1998; Chitturi and Farrell, 2001), due to increased rates of fatty acid oxidation in both mitochondria and peroxisomes, as consequence of fat accumulation.

The increase in the lag phase and the decrease in the ADP/O ratio after 16 weeks of CDD feeding indicated an uncoupling between the phosphorylative and oxidative mitochondrial subsystems. However, the decrease in the phosphorylative efficiency in 16 weeks CDD-fed animals, was not due to decreased activity or content of ATP synthase, but was caused by a depletion in ANT, as previously reported on a genetic model of NAFLD (Teodoro et al., 2006). ANT, the most abundant protein in the mitochondrial inner membrane, is essential to oxidative phosphorylation by exchanging ADP and ATP, thus maintaining ATP cytosolic content.

The analysis of the hepatic mitochondrial function 12 weeks CDD-fed animals revealed an extremely interesting response, trying to revert the deleterious effects of fat accumulation. Despite some of the data demonstrate the continuity of the aggression of the fat on the mitochondrial functionality (maintenance of the macrovesicular steatosis associated with increased state 4 , increased respiratory rate in the presence of oligomycin and increased susceptibility to MPT induction), most of the evaluated parameters totally reverted their tendency towards impairment of mitochondrial function. In fact, the increased state 3, FCCP-induced oxygen consumption and enzymatic activities (succinate dehydrogenase, cytochrome $c$ oxidase, and ATP synthase), when compared to control animals, suggest a recovery on most of the mitochondrial functionality that was lost, as demonstrated by the data from the 4 and 8 weeks CDD-fed animals. The decreased lag phase and the unaltered $\mathrm{ADP} / \mathrm{O}$ demonstrated that mitochondrial oxidative and phosphorylative subsystems were perfectly coupled by 12 weeks of feeding CDD diet. Furthermore, the analysis of the ANT content on the CDD 12 weeks revealed a tendency, although not statistically significant, to an increased ANT content on these animals; no alterations were found on the content on ATP synthase. Curiously, the data from the CDD animals after 12 weeks of feeding demonstrated a probable increased redox capability, as suggested by the decrease in protein carbonyl content, comparatively to the same age control animals.

These data clearly suggest that fat accumulation causes a severe impairment of mitochondrial function, but more importantly, it also triggers a response mechanism, designed to withstand (and probably overcome) the challenge created by the steatosis, on the bioenergetic level. While this manuscript was in preparation, work conducted by Petrosillo and co-workers demonstrated that impairment of complex I activity after 30 weeks of feeding with a choline-deficient diet is caused by a ROS dependent cardiolipin peroxidation (Petrosillo et al., 2007). Our study confirms that the mitochondrion plays a central role in the development of the non-alcoholic steatosis, particularly on the oxidative phosphorylation efficiency level. Moreover, by studying the progression of the pathology through 16 weeks of choline-deficient diet, it unveiled an extremely interesting response designed to prevent and revert the mitochondrial damage associated with this pathology. Further studies on the development and physiological mechanisms of nonalcoholic fatty liver, specially aimed at the regulation of mitochondrial function, are required in order to fully understand this disease and unravel possible therapeutic targets.

\section{Acknowledgements}

We acknowledge the Laboratório de Análises Clínicas-FFUC for plasma biochemical analysis, as well as Raquel Seiça and the Serviço de Anatomia Patológica-HUC for liver histology.

\section{References}

Baffy, G., Zhang, C.Y., Glickman, J.N., Lowell, B.B., 2002. Obesity-related fatty liver is unchanged in mice deficient for mitochondrial uncoupling protein 2 . Hepatology 35, 753-761.

Begriche, K., Igoudjil, A., Pessayre, D., Fromenty, B., 2006. Mitochondrial dysfunction in NASH: causes, consequences and possible means to prevent it. Mitochondrion 1, 1-28.

Brautigan, D.L., Ferguson-Miller, S., Margoliash, E., 1978. Mitochondrial cytochrome $c$ : preparation and activity of native and chemically modified cytochrome $c$. Methods in Enzymology 53, 128-133.

Brealey, D., Brand, M., Hargreaves, I., Heales, S., Land, J., Smolenski, R., Davies, N.A., Cooper, C.E., Singer, M., 2002. Association between mitochondrial dysfunction and severity and outcome of septic shock. Lancet 360, 219-223.

Browning, J.D., Horton, J.D., 2004. Molecular mediators of hepatic steatosis and liver injury. The Journal of Clinical Investigation 114, 147-152.

Caldwell, S.H., Chang, C.Y., Nakamoto, R.K., Krugner-Higby, L., 2004. Mitochondria in non-alcoholic fatty liver disease. Clinics in Liver Disease 8, 595-617.

Chitturi, S., Farrell, G.C., 2001. Etiopathogenesis of nonalcoholic steatohepatitis. Seminars in Liver Disease 21, 27-41.

Clark, J.M., Diehl, A.M., 2003. Nonalcoholic fatty liver disease: an underrecognized cause of cryptogenic cirrhosis. The Journal of the American Medical Association 289, 3000-3004

Danzani, M.U., 1957. Uncoupling of oxidative phosphorylation in mitochondria from fatty livers. The Biochemical Journal 65, 116-124.

Day, C.P., James, O.F., 1998. Hepatic steatosis: innocent bystander or guilty party? Hepatology 27, 1463-1466.

DiMauro, S., Schon, E.A., 2003. Mitochondrial respiratory-chain diseases. The New England Journal of Medicine 348, 2656-2668.

Diraison, F., Yankah, V., Letexier, D., Dusserre, E., Jones, P., Beylot, M., 2003. Differences in the regulation of adipose tissue and liver lipogenesis by carbohydrates in humans. Journal of Lipid Research 44, 846-853.

Donthamsetty, S., Bhave, V.S., Mitra, M.S., Latendresse, J.R., Mehendale, H.M., 2007. Nonalcoholic fatty liver sensitizes rats to carbon tetrachloride hepatotoxicity. Hepatology 45, 391-403.

Douette, P., Navet, R., Gerkens, P., de Pauw, E., Leprince, P., Sluse-Goffart, C., Sluse, F.E., 2005. Steatosis-induced proteomic changes in liver mitochondria evidenced by two dimensional differential in-gel electrophoresis. Journal of Proteome Research 4, 2024-2031.

Duchen, M.R., 2004. Mitochondria in health and disease: perspectives on a new mitochondrial biology. Molecular Aspects of Medicine 25, 365-451.

Estabrook, R.W., 1967. Mitochondrial respiratory control and the polarographic measurements of ADP/O ratios. Methods in Enzymology 10, 41-47.

Fromenty, B., Pessayre, D., 1997. Impaired mitochondrial function in microvesicular steatosis. Effects of drugs, ethanol, hormones and cytokines. Journal of Hepatology 26, 43-53.

Gazotti, P., Malmstrom, K., Brdiczka, D., 1979. Membrane Biochemistry: A Laboratory Manual on Transport and Bioenergetics. Springer-Verlag, New York. pp. 62-69.

Gornall, A.G., Bardawill, C.J., David, M.M., 1949. Determination of serum proteins by means of the biuret reaction. The Journal of Biological Chemistry 177, 751-766.

Grattagliano, I. Caraceni, P., Portincasa, P. Domenicali, M., Palmieri, V.O., Trevisani, F., Bernardi, M., Palasciano, G., 2003. Adaptation of subcellular glutathione detoxification system to stress conditions in choline-deficient diet induced rat fatty liver. Cell Biology and Toxicology 19, 355-366.

Haque, M., Sanyal, A., 2002. The metabolic abnormalities associated with nonalcoholic fatty liver disease. Best Practice \& Research, Clinical Gastroenterology 16, 709-731.

Jacobsen, E.B., Hamberg, O., Quisorff, B., Ott, P., 2001. Reduced mitochondrial adenosine triphosphate synthesis in skeletal muscle in patients with ChildPugh Class B and C cirrhosis. Hepatology 34, 7-12.

Kamo, N., Muratsugu, M., Hongoh, R., Kobatake, V., 1979. Membrane potential of mitochondria measured with an electrode sensitive to tetraphenyl phosphonium and relationship between proton electrochemical potential and phosphorylation potential in steady state. The Journal of Membrane Biology 49, 105-121.

Koteish, A., Diehl, A.M., 2001. Animal models of steatosis. Seminars in Liver Disease 21, 89-104

Leclercq, I.A., Farrell, G.C., Field, J., Bell, D.R., Gonzalez, F.J., Robertson, G.R., 2000. CYP2E1 and CYP4A as microsomal catalysts of lipid peroxides in murine nonalcoholic steatohepatitis. The Journal of Clinical Investigation 105, 1067-1075.

Levine, R.L., Garland, D., Oliver, C.N., Amici, A., Climent, I., Lenz, A.G., Ahn, B.W., Shaltiel, S., Stadtman, E.R., 1990. Determination of carbonyl content in oxidatively modified proteins. Methods Enzymology 186, 464-478.

Mahler, H., Pasi, A., Kramer, J.M., Schulte, P., Scoging, A.C., Bar, W., 1997. Fulminant liver failure in association with the emetic toxin of Bacillus cereus. The New England Journal of Medicine 336, 1142-1148.

McClain, C.J., Mokshagundam, S.P., Barve, S.S., Song, Z., Hill, D.B., Chen, T., Deaciuc, I., 2004. Mechanisms of non-alcoholic steatohepatitis. Alcohol 34, 67-79.

Oliveira, P.M.S., Gayotto, L.C., Tatai, C., Bina, B.I.D., Janiszewski, M., Lima, E.S., Abdalla, E.S.P. Lopasso, F.P., Laurindo, F.R.M., Laudanna, A.A., 2002. Oxidative stress in the pathogenesis of non-alcoholic fatty liver disease in rats fed with a choline-deficient diet. Journal of Cellular and Molecular Medicine 6, 399-406.

Palmeira, C.M., Moreno, A.J., Madeira, V.M.C., 1994. Interactions of herbicides 2,4-D and dinoseb with liver mitochondrial bioenergetics. Toxicology and Applied Pharmacology 127, 50-57. 
Palmeira, C.M., Ferreira, F.M., Rolo, A.P., Oliveira, P.J., Santos, M.S., Moreno, A.J., Cipriano, M.A., Martins, M.I., Seiça, R., 2003. Histological changes and impairment of liver mitochondrial bioenergetics after long-term treatment with $\alpha$-naphthyl-isothiocyanate (ANIT). Toxicology 190, 185-196.

Palmeira, C.M., Wallace, K.B., 1997. Benzoquinone inhibits the voltage-dependent induction of the mitochondrial permeability transition caused by redox-cycling naphthoquinones. Toxicology and Applied Pharmacology 143, 338-347.

Pessayre, D., Mansouri, A., Fromenty, B., 2002. Nonalcoholic steatosis and steatohepatitis. Mitochondrial dysfunction in steatohepatitis. American Journal of Physiology, Gastrointestinal and Liver Physiology 282, G193-G199.

Petrosillo, G., Portincasa, P., Grattagliano, I., Casanova, G., Matera, M., Ruggiero, F.M., Ferri, D., Paradies, G., 2007. Mitochondrial dysfunction in rat with nonalchoolic fatty liver. Involvemnt of complex I, reactive oxygen species and cardiolipin. Biochimica et Biophysica Acta 1767, 1260-1267.

Rao, M.S., Reddy, J.K., 2001. Peroxisomal beta-oxidation and steatohepatitis. Seminars in Liver Disease 21, 43-55.

Sayeed, M.M., 2002. Mitochondrial dysfunction in sepsis: a familiar song with new lyrics. Critical Care Medicine 30, 2780-2781.

Schatz, G., 1995. Mitochondria: beyond oxidative phosphorylation. Biochimica et Biophysica Acta 271, 123-126.
Schild, L., Reinheckel, T., Wiswedel, I., Augustin, W., 1997. Short-term energy production in isolated rat mitochondria by hypoxia/reoxygenation: involvement of oxidative protein modification. The Biochemical Journal 328, 205-210.

Singer, T.P., 1994. Determination of the activity of succinate, NADH, choline and glycerophosphate dehydrogenases. Biochimica et Biophysica Acta 1363, 100 124.

Srere, P.A., 1969. Citrate synthase. Methods in Enzymology, vol. 13. Academic Press, New York. pp. 3-11.

Teodoro, J., Rolo, A.P., Oliveira, P.J., Palmeira, C.M., 2006. Decreased ANT content in Zucker fatty rats: Relevance for altered mitochondrial bioenergetics in steatosis. FEBS Letters 580, 2153-2157.

Vendemiale, G., Grattagliano, I., Caraceni, P., Caraccio, G., Domenicali, M., Dall'Agata M., Trevisani, F., Guerrieri, F., Bernardi, M., Altomare, E., 2001. Mitochondrial oxidative injury and energy metabolism alteration in rat fatty liver: effect of the nutritional status. Hepatology 33, 808-815.

Zafrani, E., 2004. Non-alcoholic fatty liver disease: an emerging pathologica spectrum. Virchows Archiv: An International Journal of Pathology 444, 3-12.

Zeisel, S.H., Blusztajn, J.K., 1994. Choline and human nutrition. Annual Review of Nutrition 14, 269-296. 